\title{
Actions of prolonged glucagon-like peptide-1 receptor activation on cognitive function in a model of diet-induced obesity
}

\author{
D. W. Porter, B. D. Kerr, P. R. Flatt, C. Holscher and V. A. Gault \\ The SAAD Centre for Pharmacy and Diabetes, School of Biomedical Sciences, University of Ulster, Coleraine, \\ Northern Ireland, UK
}

Liraglutide $\left(\right.$ Victoza $^{\circledR}$ ) is a once-daily glucagon-like peptide-1 (GLP-1) mimetic currently prescribed as a therapy for type-2 diabetes ${ }^{(1)}$. Liraglutide mimics all of the glucoregulatory, insulin-releasing and extra-pancreatic actions of GLP-1, especially the glucose-dependent stimulation of insulin secretion ${ }^{(2)}$. Recent studies have shown that Liraglutide crosses the blood-brain-barrier when administered peripherally $^{(3)}$ and GLP-1R deficient mice exhibit impaired memory and learning ${ }^{(4)}$. Therefore, the present study examined the effects of daily treatment with Liraglutide on the cognitive function in an animal model of diet-induced obesity which exhibits compromised cognitive performance.

Young Swiss TO mice (6-8 weeks old; $n 10$ per group) maintained on high-fat diet (45\% fat, $20 \%$ protein and $35 \%$ carbohydrate) for 20 weeks received twice-daily injections of Liraglutide $(200 \mu \mathrm{g} / \mathrm{kg}$ bw; $s c)$ or saline vehicle over $28 \mathrm{~d}$. An additional group of mice on standard diet (10\% fat, $30 \%$ protein, $60 \%$ carbohydrate) received twice-daily saline injections. Energy intake, bodyweight and plasma glucose and insulin concentrations were monitored at regular intervals. Glucose tolerance, open field assessment, object recognition testing and electrophysiological long-term potentiation (LTP) were performed at the termination of the study.

Treatment with Liraglutide significantly reduced bodyweight $(1.1$-fold; $P<0.05)$ and energy intake $(1.5$-fold; $P<0.01)$, while improving non-fasting glucose $(50-230 \%$ reduction; $P<0.01$ to $P<0.001)$, insulin (30-50\% increase; $P<0.05$ to $P<0.01)$ and normalising glucose tolerance $(40-50 \%$ improvement; $P<0.05)$ compared to high fat controls. During the object recognition trial, mice on high-fat diet demonstrated a significant decrease in recognition index (RI), whereas mice treated with Liraglutide exhibited a significant increase in RI (1.4-fold; $P<0.05)$ indicative of enhanced memory and learning ability. Interestingly, the RI for Liraglutide-treated mice was broadly similar to that observed for healthy age-matched normal mice, highlighting a reversal in the cognitive decline following Liraglutide treatment in this model. In vivo hippocampal LTP was completely abolished following high-fat diet. However, daily treatment with Liraglutide ameliorated $(P<0.001$ to $P<0.0001)$ the detrimental effects of high-fat diet on LTP formation and maintenance.

In conclusion, this study demonstrates that prolonged GLP-1R activation with Liraglutide exhibits beneficial effects on the cognitive function and hippocampal synaptic plasticity in a mouse model of high-fat diet-induced obesity. Given the increasing awareness of a negative impact of obesity-diabetes on brain function, possible protective effects of GLP-1 mimetics on cognitive parameters need to be assessed in the rising numbers of obese type- 2 diabetes patients taking incretin therapeutics.

1. Knudsen LB (2004) J Med Chem 47, 4128-4134.

2. Lovshin JA \& Drucker DJ (2009) Nat Rev Endocrinol 5, 262-269.

3. McClean PL, Gault VA, Harriott P et al. (2010) Eur J Pharmacol 630, 158-162.

4. Abbas T, Faivre E \& Holscher C (2009) Behav Brain Res 205, 265-271. 